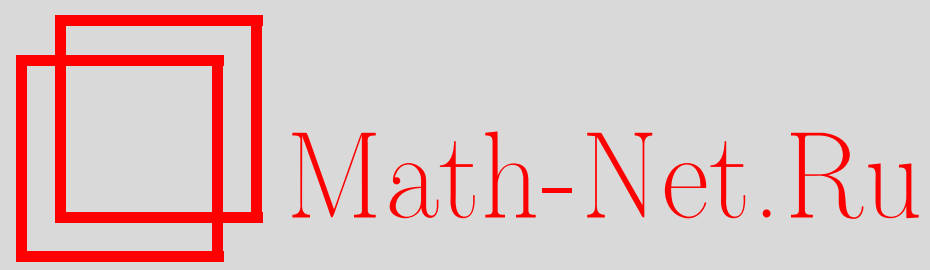

И. Ю. Фёдоров, О некоторых расслоениях на коники, $M a-$ тем. заметки, 1998, том 63, выпуск 6, 903-910

DOI: https://doi.org/10.4213/mzm1361

Использование Общероссийского математического портала Math-Net.Ru подразумевает, что вы прочитали и согласны с пользовательским соглашением http://www.mathnet.ru/rus/agreement

Параметры загрузки:

IP : 3.89.185.249

26 апреля 2023 г., 14:43:38

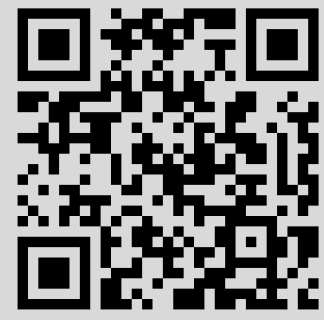




\title{
О НЕКОТОРЫХ РАССЛОЕНИЯХ НА КОНИКИ
}

И. Ю. Фёдоров

\begin{abstract}
Описана реализация антиканонической К3 поверхности особой связки коник, обобщающая известную теорему Шокурова.

Библиографиия: 9 названий.
\end{abstract}

1. Введение. Пусть $X$-проективное трехмерное многообразие. Тогда если $\varkappa(X)=$ $-\infty$, то $X$ бирационально эквивалентно $\mathbb{Q}-\Phi$ ано расслоенному пространству, т.е. многообразию $X^{\prime}$ с $\mathbb{Q}$ - факториальными терминальными особенностями, имеющему проективный морфизм $p: X^{\prime} \rightarrow Y$ на нормальное проективное многообразие $Y, \rho(X / Y)=1$, со связньми слоями такой, что $-K_{X}$ является $p$-обильньп дивизором и $\operatorname{dim} Y \leqslant 2$ (см. [1]).

В гладком случае классификацию многообразий с $-K_{X}$ обильным, удовлетворяющих условию $\operatorname{Pic} X=\mathbb{Z}$, осуществил В.А. Исковских (см. [2]), используя следующий результат В.В. Шокурова.

Tеорема [3]. Пусть $X$ - неособое многообразие Фано индекса Фано $r \geqslant 1$ и $H \in \operatorname{Pic} X-$ дивизор такой, что $-K_{X}=r H$. Тогда в линейной системе $\left|\mathscr{O}_{X}(H)\right|$ существует гладкая поверхность.

Позднее М. Рид высказал знаменитую гипотезу о слоне, имеющую важное значение в современной бирациональной геометрии.

ГИПоТЕЗА о СЛонЕ. Пусть $X$ - нормальное трехмерное многообразие с терминальными $\mathbb{Q}$-факториальными особенностями, $p: X \rightarrow S-$ проективный морфизм, $p_{*} \mathscr{O}_{X}=\mathscr{O}_{S}$ и дивизор $-K_{X}$ является $p$-обильным. Тогда общий әлемент линейной системы $\left|-K_{X}+p^{*}(A)\right|$, где $A$ - достаточно обильный дивизор Картье на $S$, имеет лишь дювалевские особенности (так называемый дювалевский слон).

В своей работе [1] Рид доказал обобщение теоремы Шокурова на случай многообразия $X$, имеющего канонические особенности, $-K_{X} \equiv Q$, где $Q$ - дивизор Картье. В [4] Ю.Г. Прохоров обобщил теорему из [5] на лог-терминальный случай. В настоящей работе исследуется существование хорошего слона, т.е. слона с лог-терминальными особенностями, на $\mathbb{Q}-\Phi$ ано расслоенных пространствах - расслоениях на коники.

Всюду далее $X$ - трехмерное расслоение на коники с лог-терминальными особенностями, $p: X \rightarrow S$ - проективньй морфизм на нормальную поверхность $S, A$ - достаточно обильньй дивизор на $S, H=p^{*} A-Q$.

Работа выполнена при финансовой поддержке Российского фонда фундаментальных исследований, грант № 96-01-00820, и фонда INTAS, грант № 93-2805. 
Теорема 1. Пусть $-K_{X} \equiv Q$, где $Q-$ - дивизор Картье. Тогда линейная система $|H|=\left|p^{*} A-Q\right|$ содержит приведенный неприводимый дивизор лишь $c$ лог-терминальными особенностями.

Теорема 1 будет доказана в п. 3. В п. 2 приведены примеры таких многообразий.

2. Примеры. В дальнейшем считаем, что все многообразия определены над $\mathbb{C}$. Необходимые определения содержатся в [6]-[8].

Построение лог-терминальных расслоений на коники будем производить локально. Рассмотрим фактор $Y / G, Y=\mathbb{C}^{2} \times \mathbb{P}^{1}$, по групе $G$, действующей по правилу $x \mapsto e^{a} x$, $y \mapsto e^{b} y$ и $z \mapsto-z$ в соответствуюшей карте на $\mathbb{P}^{1}$. Порядок групшы $G$ равен $2 p$, где $p>3$ - простое число, $a, b$ - взаимно простые числа, не делящиеся на $p$. Тогда $X$ имеет только лог-терминальные особенности (см. [6, утверждение 6.7]). Докажем, что $K_{X} \equiv Q$ где $Q$ - дивизор Картье. Для этого необходимо построить инвариантную дифференциальную форму. При $a+b \equiv p \bmod 2 p$ такой инвариантной формой будет $d x \wedge d y \wedge d z$, так как ее вес $\operatorname{wt}(d x \wedge d y \wedge d z)=a+b-p$ равен 0 . В другой карте эта форма записывается, как $z^{-2} d x \wedge d y \wedge d z$, и ее вес равен $2 p$. Отметим, что $K_{X} \cdot Z=-1$, где $Z$ - цикл над $0 \in \mathbb{C}^{2}$.

\section{3. Существование хорошего дивизора.}

ПРЕДЛОЖЕНИЕ 1. Для достаточно больиих A система $\mid$ Н $\mid$ непуста.

ДокАЗАТЕЛЬСТво. Выберем обильньй дивизор $W$ на поверхности $S$ и положим $A=l W$, где $l \gg 0$. По теореме Римана-Роха

$$
\chi\left(\mathscr{O}_{x}(H)\right)=\frac{3}{4} l^{2}\left(p^{*} W\right)^{2} \cdot\left(-K_{X}\right)+\cdots>0 .
$$

По теореме Каваматы-Фивега об обрашении в нуль (см. [7, теорема $1-2-5]) h^{j}(\mathscr{O}(H))=0$ для $i>0, l \gg 0$. Следовательно, $h^{0}\left(\mathscr{O}_{X}(H)\right)>0$, что и требовалось доказать.

Заметим, что если $B_{S}|H|=\varnothing$, то по теореме Бертини и формуле присоединения общий дивизор из системы $|H|$ имеет лишь лог-терминальные особенности (см., например, $\left[6\right.$, лемма 6.6]). Таким образом, мы можем считать, что $B_{S}|H| \neq \varnothing$.

Воспользуемся конструкцией Каваматы, согласно которой существуют разрешение $f: Y \rightarrow X$ и дивизор $\sum E_{i}$ на $Y$ с простыми нормальными пересечениями такие, что

i) $K_{Y} \equiv f^{*} K_{X}+\sum a_{i} E_{i}$, где $a_{i} \in \mathbb{Q}, a_{i}>-1$ и $a_{i} \neq 0$ только в случае $f_{*} E_{i}=0$;

ii) $f^{*}|H|=|L|+\sum r_{i} E_{i}$, где $|L|$ - свободная линейная система на $Y, r_{i} \in \mathbb{Z}, r_{i} \geqslant 0$ и $r_{i}>0$ по крайней мере для одного индекса $i$;

iii) для любого $\varepsilon>0$ найдутся $p_{i}, q \in \mathbb{Q}, 0<p_{i} \ll q \ll \varepsilon$, такие, что $\mathbb{Q}$-дивизор $q f^{*} H-\sum p_{i} E_{i}$ обилен;

iv) $f$ является 0-минимальньм разрешением в смысле [5, теоремы 4.1-4.3], т.е. $f$ индуцирует минимальное хорошее разрешение на общем гиперплоском сечении $X^{\prime} \subset X$ и $\left|H^{\prime}\right|:=|H|_{X^{\prime}}$.

ЗАмЕЧАниЕ (cp. с [5, теорема 4.2]). Согласно iv) имеем следующее:

1) если $\operatorname{dim} f\left(E_{i}\right) \neq 0$, то $r_{i} \geqslant a_{i}$;

2) если $\operatorname{dim} f\left(E_{i}\right) \neq 0$, то $r_{i}>a_{i}$ за исключением случаев

a) $\operatorname{dim} f\left(E_{i}\right)=1$, многообразие $X$ неособо в общей точке $f\left(E_{i}\right), f$-раздутие $f\left(E_{i}\right)$ и $r_{i}=a_{i}=1$, 
б) $\operatorname{dim} f\left(E_{i}\right)=1, X$ имеет лиш $\mathrm{cDV}$-особенность в общей точке $f\left(E_{i}\right), f\left(E_{i}\right) \notin$ $B_{S}|H|$ и $r_{i}=a_{i}=0$.

Лемма 1. Следующие два условия әквивалентны:

1) $a_{i}+1>r_{i}$ для всех $i$

2) общий дивизор линейной системы $|H|$ есть нормальная поверхность с логтерминальными особенностями.

Доказательство вытекает из приведенной конструкции.

Положим $c=\min \left\{\left(a_{i}+1-p_{i}\right) / r_{i} \mid r_{i} \neq 0\right\}$. Тогда $c \leqslant 1-p_{i} / r_{i}<1$. Слегка изменяя $p_{i}$, можно считать, что минимум достигается только для одного индекса, скажем для $i=0$. Обозначим соответствуюший дивизор через $E:=E_{0}$. Для $t \in \mathbb{Z}$ рассмотрим следуюший $\mathbb{Q}$-дивизор на многообразии $Y$ :

$$
\begin{aligned}
N & =N(t)=t f^{*} H+\sum\left(-c r_{i}+a_{i}-p_{i}\right) E_{i}-K_{Y} \\
& \equiv c L+f^{*}(t-c+1) H-\sum p_{i} E_{i}-f^{*} p^{*} A \equiv(t-c) f^{*} H-f^{*} K_{X}+c L-\sum p_{i} E_{i} .
\end{aligned}
$$

Дивизор $N(t)$ обилен при $t \geqslant 1,(p \circ f)$-обилен при $t-c+1 \geqslant q>0$, и его дробная часть содержится в $\sum E_{i}$. Для верхней целой части $N$ имеем $\lceil N\rceil=t f^{*} H-K_{Y}+B-E$, где $B$ - эффективньй дивизор, состоящий из компонент $E_{i}$, исключительных для $f$.

ПРЕДЛОЖЕНИЕ 2. Если $t \geqslant c+q-1, t \neq 0, i>0$, mo

1) $H^{i}\left(E, \mathscr{O}_{E}\left(t f^{*} H+B\right)\right)=0$;

2) $H^{i}\left(Y, \mathscr{O}_{Y}\left(t f^{*} H+B-E\right)\right)=0$.

ДокАЗАТЕЛЬСТво. Из теоремы Каваматы-Фивега следует, что

$$
0=R^{i}(f p)_{*}\left(t f^{*} H+B-E\right)=H^{i}\left(Y, \mathscr{O}_{Y}\left(t f^{*} H+B-E+f^{*} p^{*} L^{\prime}\right)\right)
$$

где $L^{\prime}$ - достаточно обильньй дивизор на $S$. Согласно выбору дивизора $A$ это доказывает утверждение 2).

Утверждение 1) является очевидным следствием утверждения 2).

ПРЕДЛОЖЕНИЕ 3. 1) Если $c \leqslant 2-q, \operatorname{mo~}^{0}\left(\mathscr{O}_{E}\left(f^{*} H+B\right)\right)=0$.

2) Если $c \leqslant 1-q$, mo $h^{0}\left(\mathscr{O}_{E}(B)\right) \geqslant 1$.

ДокАЗАТЕЛЬСТво. Утверждение 1) следует из предложения 2 и точной последовательности

$$
0 \longrightarrow \mathscr{O}_{Y}\left(f^{*} H+B-E\right) \longrightarrow \mathscr{O}_{Y}\left(f^{*} H+B\right) \longrightarrow \mathscr{O}_{E}\left(f^{*} H+B\right) \longrightarrow 0
$$

Так как $E$ не лежит в носителе $B$, получаем утверждение 2 ).

Следуя работам [5], [9], [4], докажем теорему 1 , предполагая, что $a_{i}+1 \leqslant r_{i}$ для некоторого $i$. Это приведет нас к противоречию с тем, что $h^{0}\left(\mathscr{O}_{E}\left(f^{*} H+B\right)\right)=0$.

ЛЕмма 2. Если $f(E)-$ точка, то $c>2-q$. 
ДокАЗАТЕЛЬСТво. Предположим, что $c \leqslant 2-q$. Поскольку $f(E)$ - точка, имеем

$$
\chi\left(\mathscr{O}_{E}\left(t f^{*} H+B\right)\right)=\chi\left(\mathscr{O}_{E}(B)\right),
$$

и согласно предложению $3 \chi\left(\mathscr{O}_{E}(B)\right)=h^{0}\left(\mathscr{O}_{E}\left(t f^{*} H+B\right)\right)$ при $t \geqslant 1$. Таким образом, $h^{0}\left(\mathscr{O}_{Y}\left(t f^{*} H\right)\right)>0$ при $t \gg 0$, откуда $\chi\left(\mathscr{O}_{E}(B)\right)>0$. Последнее противоречит утверждению 1 ) предложения 3 , согласно которому $\chi(\mathscr{O}(B))=0$. Лемма доказана.

СлЕДСТВИЕ 1. Ecли $f(E)-$ точка, mо $a_{i}+1 \geqslant 2 r_{i}$ для любого $i$.

ДоКАЗАТЕЛЬСТво. Согласно лемме 2

$$
\frac{a_{i}+1-p_{i}}{r_{i}} \geqslant c>2-q, \quad a_{i}+1 \geqslant 2 r_{i}-q r_{i}+p_{i}>2 r_{i}-\varepsilon .
$$

Выбирая $\varepsilon$ достаточно малым, получим $a_{i}+1 \geqslant 2 r_{i}$.

ПРЕДЛОЖЕНИЕ 4. Выполнено $c>1-q$.

ДокАзАтЕЛьСтво. По теореме Римана-Роха

$$
\begin{aligned}
\chi(t):=\chi\left(\mathscr{O}_{E}\left(t f^{*} H+B\right)\right)= & \frac{1}{2}\left(f^{*} H\right)^{2} \cdot E t^{2}+\frac{1}{2}\left(2 B \cdot f^{*} H \cdot E-\left(\left.f^{*} H\right|_{E}\right) \cdot K_{E}\right) t \\
& +\left(\left.B\right|_{E}\right) \cdot\left(\left.B\right|_{E}-K_{E}\right)+\chi\left(\mathscr{O}_{E}\right) .
\end{aligned}
$$

Предположим, что $c \leqslant 1-q$. Тогда согласно предложениям 2 и 3 имеем $\chi(1)=0$, $\chi(0)>0, \chi(t)=h^{0}\left(\mathscr{O}_{E}\left(t f^{*} H+B\right)\right)$ при $t \geqslant 0$. Следовательно,

$$
0>\chi(1)-\chi(0)=\frac{1}{2}\left(f^{*} H\right)^{2} \cdot E+\frac{1}{2}\left(2 B \cdot f^{*} H \cdot E-\left(\left.f^{*} H\right|_{E}\right) \cdot K_{E}\right),
$$

откуда

$$
0>f^{*} H \cdot B \cdot E+\left.f^{*} H\right|_{E} \cdot\left(\left.\left(f^{*} H+B-E-K_{Y}\right)\right|_{E}\right) .
$$

Слагаемое $f^{*} H \cdot B \cdot E$ в правой части (1) неотрицательно. Покажем, что

$$
\left(\left.f^{*} H\right|_{E}\right) \cdot\left(\left.\left(f^{*} H+B-E-K_{Y}\right)\right|_{E}\right) \geqslant 0 .
$$

Имеем

$$
\left.\left(f^{*} H+B-E-K_{Y}\right)\right|_{E}=\left\lceil\left. N(1)\right|_{E}\right\rceil=\left.N(1)\right|_{E}+\left(\left\lceil\left. N(1)\right|_{E}\right\rceil-\left.N(1)\right|_{E}\right) .
$$

Так как $N(1)$ обилен и $\lceil N(1)\rceil-N(1)$ эффективен, правая часть последнего равенства неотрицательна. Следовательно, неотрицательной является и правая часть (1). Полученное противоречие доказьвает предложение.

СлЕДСТВИЕ 2. Справедливы следующие утверждения:

1) $a_{i}+1 \geqslant r_{i} \partial \Omega_{s}$ всех $i$

2) общий дивизор линейной системы $|H|$ приведен;

3) если $a_{i}+1 \leqslant r_{i}$ для некоторого $i$, то $a_{i}+1=r_{i} u a_{0}+1=r_{0}$;

4) если общий дивизор системь $|H|$ имеет особенность вдоль кривой $Z$, то многообразие $X$ неособо в общей точке $Z$. 
Tеорема 2. Eсли $a_{0}+1=r_{0}, m o \operatorname{dim} f(E) \neq 2$.

ДокАЗАтЕльСтво. Предположим, что $\operatorname{dim} f(E)=2$. Тогда $a_{0}=0, r_{0}=1$. По формуле присоединения и лемме 2 имеем

$$
K_{E}=\left.\left.\left(K_{Y}+E\right)\right|_{E} \equiv\left(-L+\sum_{i \neq 0}\left(a_{i}-r_{i}\right) E_{i}+f^{*} p^{*} A\right)\right|_{E}
$$

где $a_{i}-r_{i}>0$, если только $f(E)$ - точка, и, следовательно, $a_{i}-r_{i}>-1$ для любого $i$. Таким образом, существует разложение $\left.K_{E} \equiv f^{*} p^{*} A\right|_{E}+R-G$, где $R, G-$ эффективные $\mathbb{Q}$-дивизоры на $E, f_{*} R=0$ и $G=\sum \gamma_{i} G_{i}, \gamma_{i} \leqslant 1$. Бирациональньй морфизм $f: E \rightarrow f(E)$ пропускается через минимальное разрешение $f: E \stackrel{\sigma}{\rightarrow} Z \stackrel{\mu}{\rightarrow} f(E)$. Поэтому $K_{Z}=\sigma_{*} K_{E} \equiv \sigma_{*} f^{*} p^{*} A+\sigma_{*} R-\sigma_{*} G$. Поскольку $K_{Z} \quad \mu$-численно эффективен и $\mu_{*} \sigma_{*} R=0$, имеем $\sigma_{*} R=0$. Положим $M:=\mu^{*}\left(\left.H\right|_{f(E)}\right)$. Используя $H^{0}\left(\mathscr{O}_{Z}(-M)\right)=H^{1}\left(\mathscr{O}_{Z}(-M)\right)=0$ (по теореме Рамануджана об обрашении в нуль) и теорему Римана-Роха, получим

$$
h^{0}\left(\mathscr{O}_{Z}\left(K_{Z}+M\right)\right)=\frac{1}{2} M\left(M+K_{Z}\right)+\chi\left(\mathscr{O}_{Z}\right) .
$$

С другой стороны,

$$
h^{0}\left(\mathscr{O}_{Z}(M)\right)+h^{0}\left(\mathscr{O}_{Z}\left(K_{Z}-M\right)\right) \geqslant \frac{1}{2} M\left(M-K_{Z}\right)+\chi\left(\mathscr{O}_{Z}\right) .
$$

Следовательно, $h^{0}\left(\mathscr{O}_{Z}\left(K_{Z}-M\right)\right)=0$, так как $K_{E}-\left.f^{*} H\right|_{E}=\left.\left(K_{Y}-f^{*} H+R\right)\right|_{E}$ и $K_{Y}-f^{*} H+E$ неэффективен $\left(K_{Z}-M\right.$ со слоями $f$ о $p$ пересекается отрицательно и не содержит $E)$. Кроме того, $h^{0}\left(\mathscr{O}_{Z}(M)\right)=0$, поскольку $h^{0}\left(\mathscr{O}_{E}\left(f^{*} H\right)\right)=0$. Окончательно, получаем

$$
0 \geqslant \frac{1}{2} M\left(M-K_{Z}\right)+\chi\left(\mathscr{O}_{Z}\right)
$$

Докажем, что

$$
\left.f^{*} p^{*} A\right|_{E}=0
$$

Рассмотрим три случая:

a) $\operatorname{dim} p f(E)=0$;

б) $\operatorname{dim} p f(E)=2$;

в) $\operatorname{dim} p f(E)=1$.

В случае а) равенство (2) очевидно.

В случае б) $\left.f^{*} p^{*} A\right|_{E}$ численно эффективен и объемен,

$$
h^{0}\left(\mathscr{O}_{E}\left(f^{*} H\right)\right)=h^{0}\left(\mathscr{O}_{E}\left(f^{*} p^{*} A-f^{*} K_{X}\right)\right)>0,
$$

что противоречит предложению 3 .

В случае в) $Z \rightarrow C$ - линейчатая поверхность (слои-коники), где $C$ - нормализация $f(E)$, а $\left.\sigma_{*}\left(f^{*} p^{*} A\right)\right|_{Z}$ - поднятие нескольких слоев. Соотношение (2) вытекает из следуюшей леммы. 


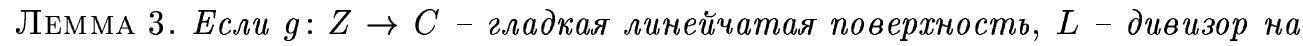

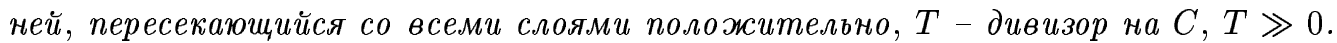
Тогда $h^{0}\left(\mathscr{O}\left(L+g^{*} T\right)\right)>0$.

ДокАзАтЕЛьство. По теореме Римана-Роха

$$
\chi\left(\mathscr{O}_{Z}\left(L+g^{*} T\right)\right)=\frac{1}{2}\left(L^{2}+2 L \cdot f^{*} T-K_{Z} \cdot L-K_{Z} \cdot g^{*} T\right)+\chi\left(\mathscr{O}_{Z}\right) .
$$

Величины $L^{2}, K_{Z} \cdot L$ и $\chi\left(\mathscr{O}_{Z}\right)$ в правой части последнего равенства постоянны. Остальные слагаемые растут вместе с ростом $T$. Поэтому для достаточно больших $T$ имеем $h^{i}\left(\mathscr{O}_{Z}\left(L+g^{*} T\right)\right)=0$ при $i>0$. Следовательно, $h^{0}\left(\mathscr{O}_{Z}\left(L+g^{*} T\right)\right)>0$.

Мы получили, что $K_{Z} \equiv-\sigma_{G}=-D$, где $D=\sum d_{i} D_{i}, 0 \leqslant d_{i} \leqslant 1$. Рассмотрим два случая:

a) $D=0, K_{Z} \equiv 0$

б) $D>0, Z$ - бирационально линейчатая поверхность над кривой $C$ рода $g(C)=$ $q(Z)=1-\chi\left(\mathscr{O}_{Z}\right) \geqslant 2$.

В случае а) $Z$ - минимальная поверхность с кодаировой размерностью $\varkappa(Z)=0$ и в соответствии с классификацией алгебраических поверхностей $\chi\left(\mathscr{O}_{Z}\right) \geqslant 0$, что противоречиво.

В случае б) имеем

$$
0 \leqslant h^{0}\left(\mathscr{O}_{Z}\left(K_{Z}+M\right)\right) \leqslant \frac{1}{2} M K_{Z}=-\frac{1}{2} M D \leqslant 0,
$$

откуда $M \cdot K_{Z}=M \cdot D=0$, т.е. $M \cdot D_{i}=0$ для любого $i$. Поэтому дивизор $D$ стягивается морфизмом $\mu$. Следовательно, $\left(D_{i}\right)^{2}<0$ и

$$
2 p_{a}\left(D_{i}\right)-2=\left(K_{Z}+D_{i}\right) \cdot D_{i}=\left(-D+D_{i}\right) \cdot D_{i}=\left(1-d_{i}\right)\left(D_{i}\right)^{2}-\sum_{j \neq i} d_{i} D_{j} \cdot D_{i} \leqslant 0
$$

откуда $p_{a}\left(D_{i}\right) \leqslant 1$. Таким образом, все компоненты $D$ содержатся в слоях морфизма $Z \rightarrow C$, и если $F$ - общий слой этого морфизма, то $D \cdot F=0$. С другой стороны, $F \simeq \mathbb{P}^{1}, F^{2}=0$ и $K_{Z} \cdot F=-D \cdot F=-2$. Противоречие.

Tеорема 3. Eсли $a_{0}+1=r_{0}, \operatorname{mo} \operatorname{dim} f(E) \neq 1$.

ДокАЗАтЕЛЬСтво. Обозначим $f(E)$ с приведенной структурой через $Z$, т.е. $Z$ приведенная неприводимая кривая. Общий дивизор системы $|H|$ имеет особенность вдоль $Z$ и согласно утверждению 4 ) следствия 2 многообразие $X$ неособо в общей точке $Z$. Поэтому $E$ получается как исключительньй дивизор раздутия (в общей точке) некоторой кривой над $Z$. Нам понадобится

Лемма 4. Если $a_{0}+1=r_{0}, \operatorname{mog} g(Z) \geqslant 2$.

ДокАЗАТЕЛЬСТво. Пусть $\nu: Z^{\prime} \rightarrow Z$ - нормализация $Z$ и $\varphi: E \rightarrow Z^{\prime}-$ соответствующий морфизм со связными слоями. Положим $D=\nu^{*}\left(\left.H\right|_{Z}\right)$. Тогда $\left.f^{*} H\right|_{E}=\varphi^{*} D$ и $\operatorname{deg} D>0$. Предположим, что $g\left(Z^{\prime}\right) \leqslant 1$. Так как $h^{0}\left(\mathscr{O}_{E}\left(f^{*} H\right)\right)=0$, имеем $h^{0}\left(\mathscr{O}_{Z^{\prime}}\left(\varphi^{*} D\right)\right)=0$. Это противоречит теореме Римана-Роха, примененной $\mathrm{k}$ кривой $Z^{\prime}$.

Таким образом, $g(Z) \geqslant 2$, что противоречит следующему утверждению. 
ПРЕДЛОЖЕНИЕ 5. Пусть $H \in|H|-$ общий дивизор. Предположсим, что поверхность Н особа вдоль кривой $Z$. Тогда $g(Z) \leqslant 1$.

ДокАЗАТЕЛЬСТво. Пусть $\widetilde{H}$ - собственньй прообраз общего дивизора системы $|H|$. Тогда

$$
\widetilde{H}=L+\sum_{\operatorname{dim} f\left(E_{i}\right)=2} r_{i} E_{i}
$$

Так как $Z \subset H$, существуют неприводимые компоненты $E_{j} \subset f^{-1}(Z)$ и $F \subset \widetilde{H}$ такие, что $f\left(E_{j} \cap F\right)=Z$. Согласно пункту 1$)$ замечания имеем $a_{j} \leqslant r_{j}$. Но из утверждения 4$)$ следствия 2 вытекает, что $X$ неособо в общей точке $Z$. Поэтому $a_{j} \in \mathbb{Z}$ и $a_{j}+1=r_{j}$.

Из формулы присоединения и леммы 2 получаем

$$
K_{F}=\left.\left.\left(K_{Y}+F\right)\right|_{F} \equiv\left(-L+\sum_{i}\left(a_{i}-r_{i}\right) E_{i}+F+f^{*} p^{*} A\right)\right|_{F}
$$

Рассмотрим три случая:

a) $F=E_{k}$ для некоторого $k, f(F)$ - неподвижная компонента $|H|$;

б) $|L|$ составлена не из пучка и $F=L$;

в) $|L|$ составлена из пучка и $F-$ компонента $L$.

В случае а) $a_{k}=0, r_{k}=1$ и $\left.K_{F} \equiv\left(-L+\sum_{i \neq k}\left(a_{i}-r_{i}\right) E_{i}+f^{*} p^{*} A\right)\right|_{F}$.

В случае б) $K_{F} \equiv \sum\left(a_{i}-r_{i}\right) E_{i}+\left.f^{*} p^{*} A\right|_{F}$.

В случае в) $L^{2} \equiv 0, l \cdot F \equiv 0, K_{F} \equiv \sum\left(a_{i}-r_{i}\right) E_{i}+\left.f^{*} p^{*} A\right|_{F}$.

Таким образом, в каждом случае, как и при доказательстве теоремы 2 , имеем численное разложение $K_{F} \equiv f^{*} p^{*} A+R-G$, где $R, G$ - эффективные $\mathbb{Q}$-дивизоры на $F$, $R=\sum \alpha_{i} R_{i}, G=\sum \beta_{i} G_{i}, \alpha_{i} \geqslant 0,0 \leqslant \beta_{i} \leqslant 1$. Более того, из замечания следует, что если $f\left(E_{i}\right)$ не является точкой, то $a_{i}-r_{i} \leqslant 0$, откуда $f_{*} R=0$. Морфизм $f: F \rightarrow f(F)$ разлагается также в композицию $f: F \stackrel{\sigma}{\rightarrow} V \stackrel{\mu}{\rightarrow} f(F)$, где $\mu: V \rightarrow f(F)$ - минимальное разрешение нормализации $f(F)$. Поэтому канонический дивизор $V$ задается формулой $K_{V}=\sigma_{*} K_{F} \equiv \sigma_{*}\left(\left.f^{*} p^{*} A\right|_{F}+\sigma_{*} R-\sigma_{*} G\right)$. Так как $K_{V}$ является $\mu$-численно эффективным дивизором, вьполнено $\sigma_{*} R=0$. Окончательно, $K_{V} \equiv \sigma_{*}\left(\left.f^{*} p^{*} A\right|_{F}-D\right)$, где $D=\sum \beta_{i} D_{i}, 0 \leqslant \beta_{i} \leqslant 1$. Для завершения доказательства предложения 5 нам понадобится

ЛЕмма 5. $p^{*}(A) \cdot Z \equiv 0$.

ДокАЗАТЕЛЬСТво. Предположим, что $p^{*}(A) \cdot Z>0$. Тогда согласно лемме 3 можно выбрать $A \gg 0$ так, чтобы $h^{0}\left(\mathscr{O}_{E}\left(\left.f^{*} H\right|_{E}\right)\right)>0$. Полученное противоречие доказывает лемму.

Из леммы 5 для $D_{j}=\sigma\left(G_{j}\right)$ находим, что

$$
2 p_{a}\left(D_{j}\right)-2=D_{j} \cdot\left(D_{j}+K_{V}\right)=D_{j} \cdot\left(D_{j}-D\right) \leqslant 0 .
$$

Поскольку $\mu\left(D_{j}\right)=f\left(E_{j} \cap F\right)=Z$, имеем $g(Z) \leqslant p_{a}\left(D_{j}\right) \leqslant 1$. Предложение 5 доказано.

Автор выражает глубокую признательность В.А. Исковских, Ю.Г. Прохорову и И. А. Чельцову за плодотворные обсуждения. 


\section{СПИСОК ЦИТИРОВАННОЙ ЛИТЕРАТУРЫ}

[1] Mori S. Flip theorem and the existence of minimal models for 3-folds // J. Amer. Math. Soc. 1988. V. 1. P. 117-253.

[2] Исковских В. А. Лекции по трехмерным алгебраическим многообразиям: Многообразия Фано. М.: Изд-во МГУ, 1988.

[3] Шокуров В. В.Гладкость общего антиканонического дивизора на многообразиях Фано // Изв. АН СССР. Сер. матем. 1979. Т. 43. С. 430-441.

[4] Прохоров Ю. Г. О трехмерных многообразиях с гиперплоскими сечениями - поверхностями Энриквеса // Матем. сб. 1995. Т. 186. №9. С. 113-124.

[5] Reid M. Projective Morphism According to Kawamata. Preprint. Warwick, 1983.

[6] Клеменс Х., Коллар Я., Мори С. Многомерная комплексная геометрия. М.: Мир, 1993.

[7] Kawamata Y., Matsuda K., Matsuki K. Introduction to the minimal model problem // Algebraic Geometry. Proc. Symp., Sendai, 1985. Adv. Stud. Pure Math. V. 10. Tokyo: Kinokuniya, 1987. P. 283-360.

[8] Alexeev V. General elephants of $\mathbb{Q}$-Fano 3-folds // Compositio Math. 1994. V. 91. № 1. P. 91-116.

[9] Alexeev V.A. Theorems about good divisors on log Fano varieties (case of index $r>n-2$ ) // Algebraic Geometry. Proc. US-USSR Symp., 1989. Lecture Notes in Math. V. 1479. Chicago (I.L.): Springer, 1991. P. 1-9.

Московский государственный университет им. М.В. Ломоносова

Поступило

E-mail : ifedorov@orc.ru 